\title{
REFORMULATION OF THE FOURIER TRANSFORMED DISCRETIZED DIFFERENTIAL EQUATIONS*
}

\author{
BY \\ BEHROOZ COMPANI - TABRIZI \\ Marathon Oil Company, Denver Research Center, Littleton, Colorado
}

\begin{abstract}
The conventional rule for differentiation by Fourier transformation of a discretized function, namely, multiplication of the Fourier transform of the function by $i k$ and a subsequent inverse Fourier transformation, was shown to be a first-order approximation to more complete rules [1]. The $i k$ differentiation formula is an exact relation for a nondiscretized function with a continuum as its independent variable. However, this rule fails, particularly when applied to nonsmooth discretized functions of high-frequency content. A consequence of this result is that Fourier-transformed differential equations must be transformed into the Fourier space not by the $i k$ differentiation rule, but by the appropriate correct differentiation rule if the differential equation is eventually to be solved through discretization. Helmholtz's equation is discussed, and the solution to the simple diffusion equation is presented using the exact and the approximate differentiation formulas and a high-frequency initial field.
\end{abstract}

Consider a function $f(x)$, discretized at $N$ points from $x=-N / 2$ to $x=N / 2$ in equal intervals of $\Delta x$. To perform Fourier transformation on this function, spatial and spectral sampling is chosen as

$$
\begin{array}{ll}
x=\mu \Delta x, & \mu=-N / 2,-N / 2+1, \ldots, 0,1,2, \ldots, N / 2, \\
k=\nu \Delta k, & \nu=-N / 2,-N / 2+1, \ldots, 0,1,2, \ldots, N / 2,
\end{array}
$$

where the spatial and spectral discretizations are related by

$$
\Delta x \Delta k=2 \pi / N
$$

which is the minimal relationship imposed on the uncertainties in any two dual spaces by the connection between their Fourier transforms.

\footnotetext{
* Received June 28, 1985.
} 
It was shown [1] that the correct global differentiation by Fourier transformation rules corresponding to central, forward, and backward differencing applied to such a discretized function are, respectively, given by

$$
\begin{aligned}
& \frac{d^{n} f(x)}{d x^{n}}=\frac{1}{(\Delta x)^{n}} F^{-1}\left\{\left[i \sin \left(\frac{2 \pi}{N \Delta k} k\right)\right]^{n} \cdot F[f(x)]\right\}, \\
& \frac{d^{n} f(x)}{d x^{n}}=\frac{1}{(\Delta x)^{n}} F^{-1}\left\{\left[\exp \left(\frac{i 2 \pi}{N \Delta k} k\right)-1\right]^{n} \cdot F[f(x)]\right\}, \\
& \frac{d^{n} f(x)}{d x^{n}}=\frac{1}{(\Delta x)^{n}} F^{-1}\left\{\left[1-\exp \left(\frac{-i 2 \pi}{N \Delta k} k\right)\right]^{n} \cdot F[f(x)]\right\},
\end{aligned}
$$

where $n$ denotes the $n$th derivative and where $F$ and $F^{-1}$ represent the forward and inverse Fourier transformation operations, respectively. These expressions should be contrasted with the expression for a nondiscretized function,

$$
\frac{d^{n} f(x)}{d x^{n}}=F^{-1}\left\{(i k)^{n} \cdot F[f(x)]\right\}
$$

Since numerical solution of partial differential equations by digital computers always involves discretization of the functions involved, the differentiation formula (7) must be abandoned and (4)-(6) must be used to transform the particular differential equation into either the frequency or wave number domains. The method is applicable to all discretized differential equations. To illustrate the point, transform the wave equation

$$
\nabla^{2} P(x, t)-\frac{1}{v^{2}} \frac{\partial^{2} P(x, t)}{\partial t^{2}}=0
$$

into the frequency domain $\omega$, which results in the Helmholtz equation

$$
\nabla^{2} P(x, \omega)+\left(\omega^{2} / v^{2}\right) P(x, \omega)=0,
$$

where $P(x, \omega)$ is the temporal Fourier transform of $P(x, t)$. However, by (4)-(6), the correct expressions for a discretized $P(x, t)$ corresponding to central, forward, and backward differencing are, respectively, given by

$$
\begin{aligned}
& \nabla^{2} P(x, \omega)+\frac{1}{v^{2}(\Delta t)^{2}} \sin ^{2}\left(\frac{2 \pi}{N \Delta \omega} \omega\right) P(x, \omega)=0 \\
& \nabla^{2} P(x, \omega)+\frac{1}{v^{2}(\Delta t)^{2}}\left[\exp \left(\frac{i 2 \pi}{N \Delta \omega} \omega\right)-1\right]^{2} P(x, \omega)=0 \\
& \nabla^{2} P(x, \omega)+\frac{1}{v^{2}(\Delta t)^{2}}\left[1-\exp \left(\frac{-i 2 \pi}{N \Delta \omega} \omega\right)\right]^{2} P(x, \omega)=0
\end{aligned}
$$

where again $t=\mu \Delta t, \omega=\nu \Delta \omega$, and $\Delta t \Delta \omega=2 \pi / N$. 
Next, the diffusion equation is considered for numerical calculations. A simple numerical method of solution is presented, and the numerical solution by using the conventional $i k$ rule is compared with the result obtained by using the central differencing rule (Eq. (4)). An ideal solution is also presented. The diffusion equation is

$$
\nabla^{2} P(x, t)=B^{2} \partial P(x, t) / \partial t,
$$

where $B$ is taken to be a constant. When transformed into the wave number domain $k$, the following expression results;

$$
-k^{2} P(k, t)=B^{2} \partial P(k, t) / \partial t,
$$

where $P(k, t)$ is the spatial Fourier transform of $P(x, t)$. However, by (4), the correct transformation corresponding to central differencing for a discretized function $P(x, t)$ is given by

$$
-\frac{1}{(\Delta x)^{2}} \sin ^{2}\left(\frac{2 \pi}{N \Delta \omega} k\right) P(k, t)=B^{2} \frac{\partial P(k, t)}{\partial t} .
$$

For this example, consider the solution to the diffusion equation using a discretized delta function as the initial field, with the fields going to zero at $\pm(N / 2) \Delta x$ at all times. Both (14) and (15) are used and the results are compared with the analytic solution.

The method of solution is as follows. The partial differentiation with respect to time is performed by forward differencing. Denoting the time difference by

$$
\Delta t=t_{n+1}-t_{n},
$$

Eq. (15) is written as

$$
-\frac{1}{(\Delta x)^{2}} \sin ^{2}\left(\frac{2 \pi}{N \Delta k} k\right) P\left(k, t_{n+1}\right)=B^{2} \frac{P\left(k, t_{n+1}\right)-P\left(k, t_{n}\right)}{\Delta t} .
$$

Solving for $P\left(k, t_{n+1}\right)$ results in the expression

$$
P\left(k, t_{n+1}\right)=\frac{P\left(k, t_{n}\right)}{\left[1+\Delta t \cdot \sin ^{2}\left(\frac{2 \pi}{N \Delta k} k\right) / B^{2} \cdot(\Delta x)^{2}\right]} .
$$

The solution algorithms. Using (18), the solution algorithm is as follows:

$$
\begin{aligned}
& P\left(k, t_{n}\right)=F P\left(x, t_{n}\right), \\
& P\left(k, t_{n+1}\right)=\frac{P\left(k, t_{n}\right)}{\left[1+\Delta t \cdot \sin ^{2}\left(\frac{2 \pi}{N \Delta k} k\right) / B^{2} \cdot(\Delta x)^{2}\right]}, \\
& P\left(x, t_{n+1}\right)=F^{-1} P\left(k, t_{n+1}\right) .
\end{aligned}
$$


Starting with the initial condition $P(x, 0)$, time stepping is then possible. Boundary conditions at $x= \pm(N / 2) \Delta x$ in the finite model can be imposed on $P\left(x, t_{n}\right)$ at every time step. If (7) is used as the differentiation formula, the solution algorithm is given by

$$
\begin{aligned}
& P\left(k, t_{n}\right)=F P\left(x, t_{n}\right), \\
& P\left(k, t_{n+1}\right)=\frac{P\left(k, t_{n}\right)}{\left[1+\Delta t \cdot k^{2} / B^{2} \cdot(\Delta x)^{2}\right]}, \\
& P\left(x, t_{n+1}\right)=F^{-1} P\left(k, t_{n+1}\right) .
\end{aligned}
$$

For simplicity, one-dimensional numerical examples are presented.

It must be noted that the author has deliberately chosen a nonsmooth incident field containing high-frequency data, since it is the high-frequency portion of a function that renders the $i k$ differentiation rule inaccurate. Also, it has been demonstrated that Fourier transformation of a nonsmooth function, such as a step or delta function, will produce the exact analytical Fourier transformation result if the conventional Fourier transformation is followed by multiplication by $\sin \left(\frac{\pi}{N} \nu\right) /\left(\frac{\pi}{N} \nu\right) .{ }^{*}$ Since a delta function is used as the initial field, this multiplication was implemented to correct the Fourier transformations.

The exact solution to the one-dimensional diffusion equation with the initial condition

$$
P(x, 0)=\delta\left(x-x_{0}\right)
$$

and the boundary conditions

$$
\lim _{x \rightarrow \pm \infty} P(x, t)=P( \pm \infty, t)=0
$$

is found by transform methods (e.g., [2]):

$$
P(x, t)=(B / \sqrt{4 \pi t}) e^{-B^{2}\left(x-x_{0}\right) / 4 t} .
$$

Numerical results and remarks. Numerical calculations were performed with $B^{2}=20$ seconds $/(\text { meter })^{2}, \Delta t=0.01$ seconds, $\Delta x=2 \pi / N$, and $N=256$. The amplitude of the discretized delta function was normalized to give a unit area when integrated over the finite domain. Since $\Delta x=2 \pi / N$, this discretized delta function is written as

$$
D\left(x-x_{0}\right)= \begin{cases}N / 2 \pi, & x=x_{0}, \\ 0, & x \neq x_{0} .\end{cases}
$$

Five time steps were taken, and the results are graphically displayed in all figures. The units on the fields $P(x, t)$ are left dimensionless in all figures and tables, and could correspond to the electric field, pressure field, etc. The Fourier transformations were done by a fast Fourier transform algorithm with a radix of 2. Exact numerical results for these calculations at $t=0.01$ seconds are displayed in Table 1 for comparison with the analytic solution.

\footnotetext{
${ }^{*}$ N. N. Bojarski, private communication.
} 
T ABLE 1. Numerical display of the data in figures (1), (2), and (3) at .01 seconds.

\begin{tabular}{|c|c|c|c|}
\hline Index & Analytic & Conventional & Reformulated \\
\hline I & $\mathrm{P}(\mathrm{I} . .01)$ & $\mathrm{P}(\mathrm{I}, .01)$ & $\mathrm{P}(\mathrm{I}, .01)$ \\
\hline 1 & $<10(-7)$ & .000979 & .009067 \\
\hline 2 & $<10(-7)$ & -.000982 & -.009094 \\
\hline 3 & $<10(-7)$ & .000990 & .009174 \\
\hline 4 & $<10(-7)$ & -.001005 & -.009309 \\
\hline 5 & $<10(-7)$ & .001025 & .009503 \\
\hline 6 & $<10(-7)$ & -.001052 & -.009758 \\
\hline 7 & $<10(-7)$ & .001086 & .010082 \\
\hline 8 & $<10(-7)$ & -.001128 & -.010482 \\
\hline 9 & $<10(-7)$ & .001179 & .010968 \\
\hline 10 & $<10(-7)$ & -.001241 & -.011551 \\
\hline 11 & $<10(-7)$ & .001314 & .012250 \\
\hline 12 & $<10(-7)$ & -.001401 & -.013084 \\
\hline 13 & $<10(-7)$ & .001504 & .014081 \\
\hline 14 & $<10(-7)$ & -.001628 & -.015275 \\
\hline 15 & $<10(-7)$ & .001776 & .016718 \\
\hline 16 & $<10(-7)$ & -.001953 & -.018461 \\
\hline 17 & $<10(-7)$ & .002173 & .020622 \\
\hline 18 & $<10(-7)$ & -.002431 & -.023245 \\
\hline 19 & $<10(-7)$ & .002783 & .026707 \\
\hline 20 & $<10(-7)$ & -.003133 & -.030761 \\
\hline 21 & $<10(-7)$ & .003863 & .037129 \\
\hline 22 & $<10(-7)$ & -.003967 & -.043087 \\
\hline 23 & $<10(-7)$ & .006667 & .058432 \\
\hline 24 & $<10(-7)$ & -.012680 & -.062704 \\
\hline 25 & $<10(-7)$ & .020253 & .116831 \\
\hline 26 & $<10(-7)$ & -.023895 & -.079232 \\
\hline 27 & $<10(-7)$ & .120353 & .340940 \\
\hline 28 & $<10(-7)$ & 289067 & .053064 \\
\hline 29 & $<10(-7)$ & .967219 & 1.430787 \\
\hline 30 & $<10(-7)$ & 2.709731 & 1.615165 \\
\hline 31 & $<10(-7)$ & 8.171390 & 7.190118 \\
\hline 32 & 12.615663 & 12.547146 & 15.849649 \\
\hline 33 & $<10(-7)$ & 8.066177 & 7.097484 \\
\hline 34 & $<10(-7)$ & 2.640408 & 1.573957 \\
\hline 35 & $<10(-7)$ & .930306 & 1.376030 \\
\hline 36 & $<10(-7)$ & .274481 & .051002 \\
\hline 37 & $<10(-7)$ & .112762 & .319227 \\
\hline 38 & $<10(-7)$ & .022144 & .072397 \\
\hline 39 & $<10(-7)$ & .018447 & .106243 \\
\hline 40 & $<10(-7)$ & -.002363 & -.056034 \\
\hline 41 & $<10(-7)$ & .005872 & .051438 \\
\hline 42 & $<10(-7)$ & -.003418 & -.037228 \\
\hline 43 & $<10(-7)$ & .003276 & .031510 \\
\hline 44 & $<10(-7)$ & -.002600 & -.025580 \\
\hline 45 & $<10(-7)$ & .002263 & .021749 \\
\hline 46 & $<10(-7)$ & -.001932 & -.018507 \\
\hline 47 & $<10(-7)$ & .001686 & .016025 \\
\hline 48 & $<10(-7)$ & -.001477 & -.013979 \\
\hline 49 & $<10(-7)$ & .001306 & .012314 \\
\hline 50 & $<10(-7)$ & -.001162 & -.010922 \\
\hline 51 & $<10(-7)$ & .001040 & .009754 \\
\hline 52 & $<10(-7)$ & -.000936 & -.008761 \\
\hline 53 & $<10(-7)$ & .000847 & .007910 \\
\hline 54 & $<10(-7)$ & -.000770 & -.007175 \\
\hline 55 & $<10(-7)$ & .000702 & .006536 \\
\hline 56 & $<10(-7)$ & -.000643 & -.005977 \\
\hline 57 & $<10(-7)$ & .000590 & .005484 \\
\hline 58 & $<10(-7)$ & -.000544 & -.005049 \\
\hline 59 & $<10(-7)$ & .000503 & .004661 \\
\hline 60 & $<10(-7)$ & -.000465 & -.004313 \\
\hline 61 & $<10(-7)$ & .000432 & .004003 \\
\hline 62 & $<10(-7)$ & -.000402 & -.003723 \\
\hline 63 & $<10(-7)$ & .000375 & .003470 \\
\hline 64 & $<10(-7)$ & -.000350 & -.003240 \\
\hline 65 & $<10(-7)$ & .000328 & .003031 \\
\hline
\end{tabular}


Fig. 1 shows the results displayed at several times using the analytic calculations based on the ideal situations of infinite domain, $x= \pm \infty$, and a pointwise localized initial field given by

$$
P(x, 0)=\delta(x-32 \Delta x) .
$$

Eq. (27) was used to calculate the fields at 128 spatial points. Exact numbers for this calculation at $t=0.01$ seconds are displayed in Table 1 for 65 points.

Figs. 2 and 3 show the results of the calculation using the algorithm defined by Eqs. (19)-(21), and Eqs. (22)-(24), respectively.

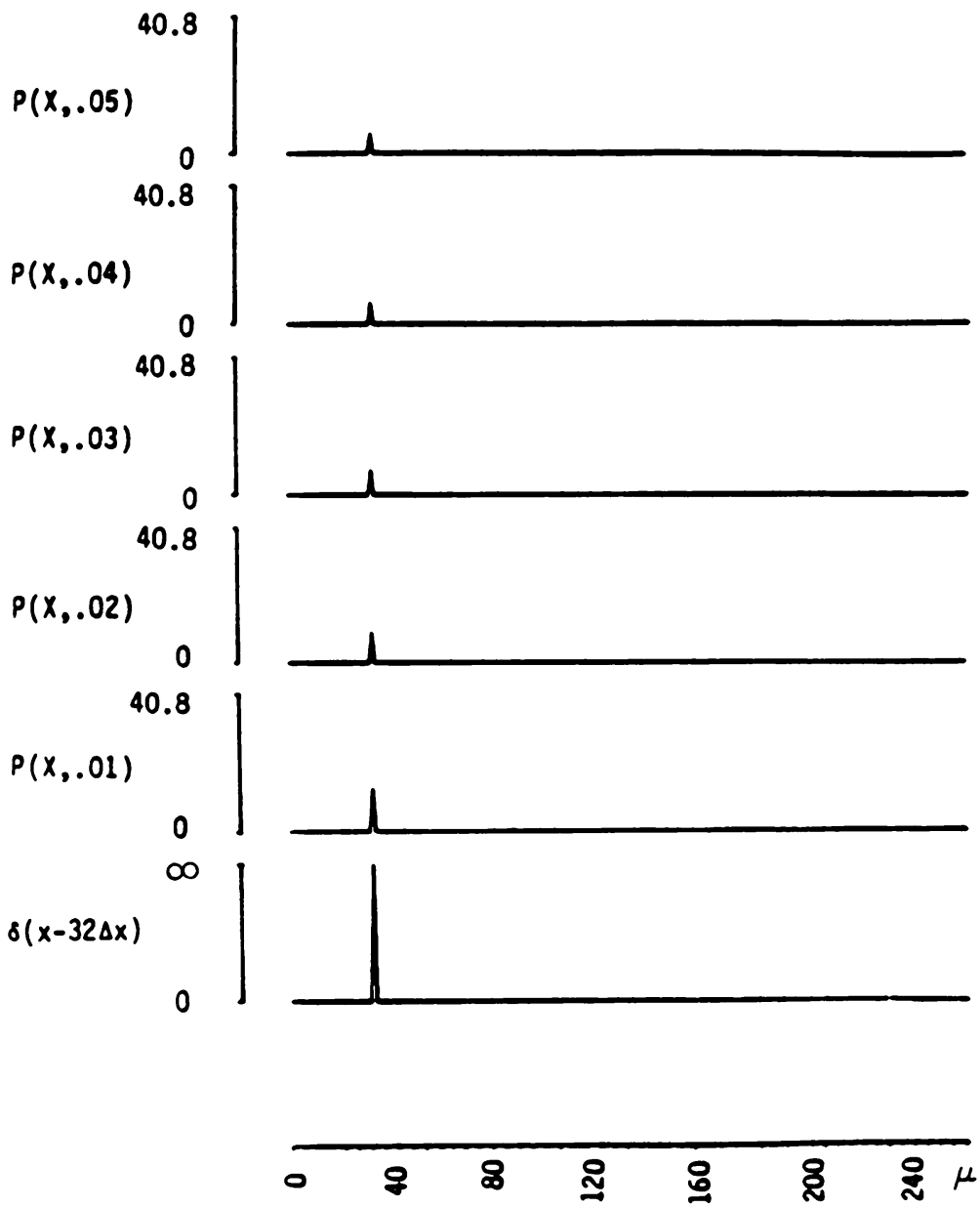

FIG. 1. Analytic solution with a delta function as the initial field. The solution is displayed at 0.01 second time intervals. 


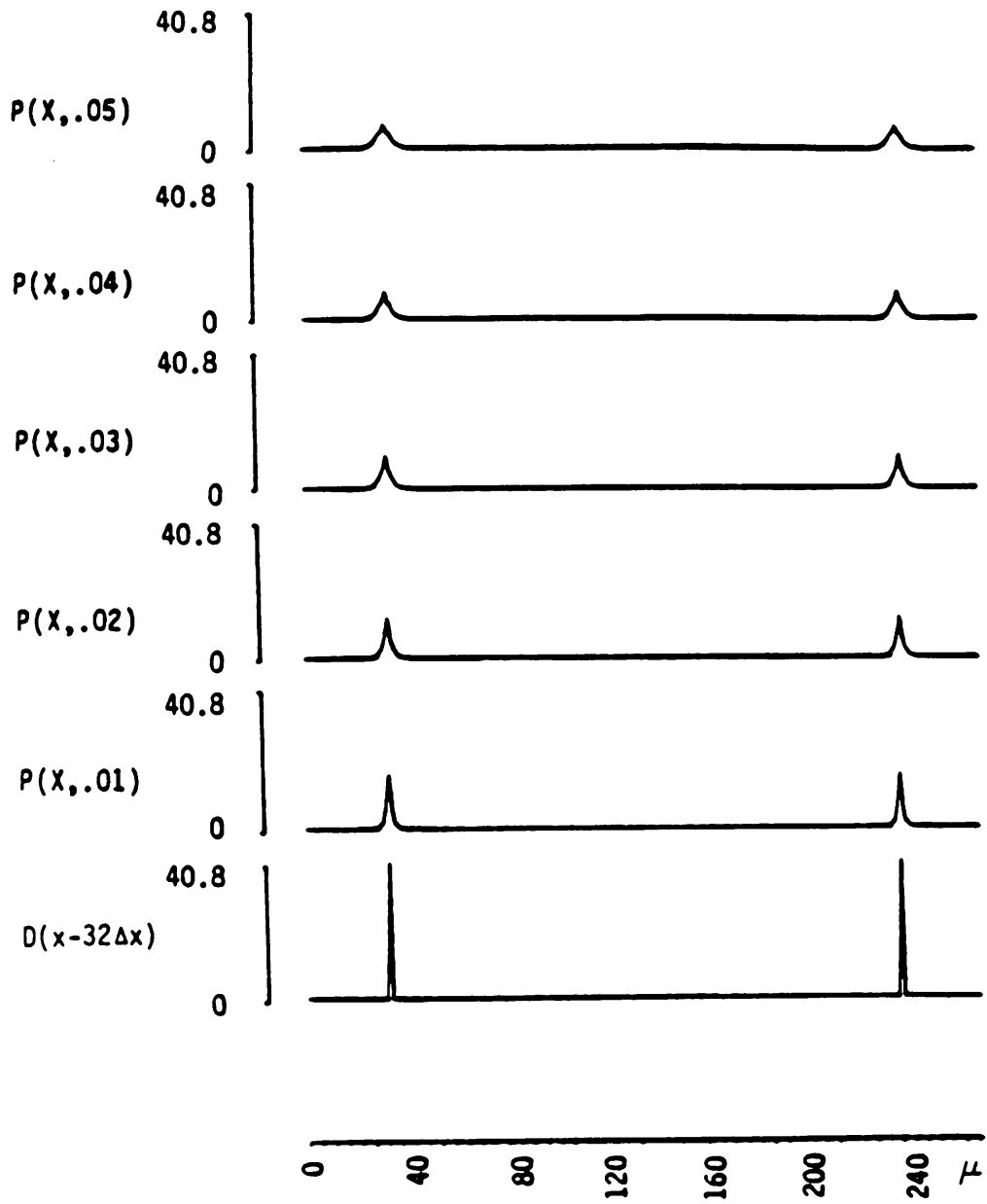

FIG. 2. Numerical solution using the reformulated discretized diffusion equation with a discretized delta function used as the initial field. The solution is displayed at 0.01 second time intervals.

From a theoretical viewpoint, the differentiation formula (4) is the correct formula to use if the numerical solution to a discretized differential equation is desired. The numerical results of this paper support this conclusion in the following sense.

(1) The analytic solution diffuses the initial fields over an infinite space $-\infty \leqslant x \leqslant+\infty$. However, the numerical solutions diffuse the initial field over a finite region. Therefore at each time, it is expected that at the peak point $(x=32 \Delta x)$ the correct numerical solution should have a higher value than the ideal analytic solution. The reformulated discretized equation results show this point.

(2) The "diffusion width-to-amplitude ratio" (i.e., the width of the field around $x=32 \Delta x$ divided by the amplitude at this point) of the results obtained using the 
reformulated discretized equation more closely approximates the "diffusion width" of the ideal solution than the results obtained using the conventional formulation.

(3) The integrated (over the finite spatial domain) field should be equal to 1 at all time steps. Although the results are close, the new approach shows a slight advantage over the old one (see Table 2).

TABLE 2. Numerical display of the integrated fields at .01 seconds time intervals.

$\begin{array}{ccc}\begin{array}{c}\text { Time } \\ \text { (Seconds) }\end{array} & \begin{array}{c}\text { Integrated Field } \\ \text { Reformulated }\end{array} & \begin{array}{c}\text { Integrated Field } \\ \text { Conventional }\end{array} \\ 0.01 & 0.9051 & 0.9049 \\ 0.02 & 0.9048 & 0.9047 \\ 0.03 & 0.9047 & 0.9046 \\ 0.04 & 0.9046 & 0.9044 \\ 0.05 & 0.9044 & 0.9043\end{array}$

Ideal Integrated Field at all times $=1.0$

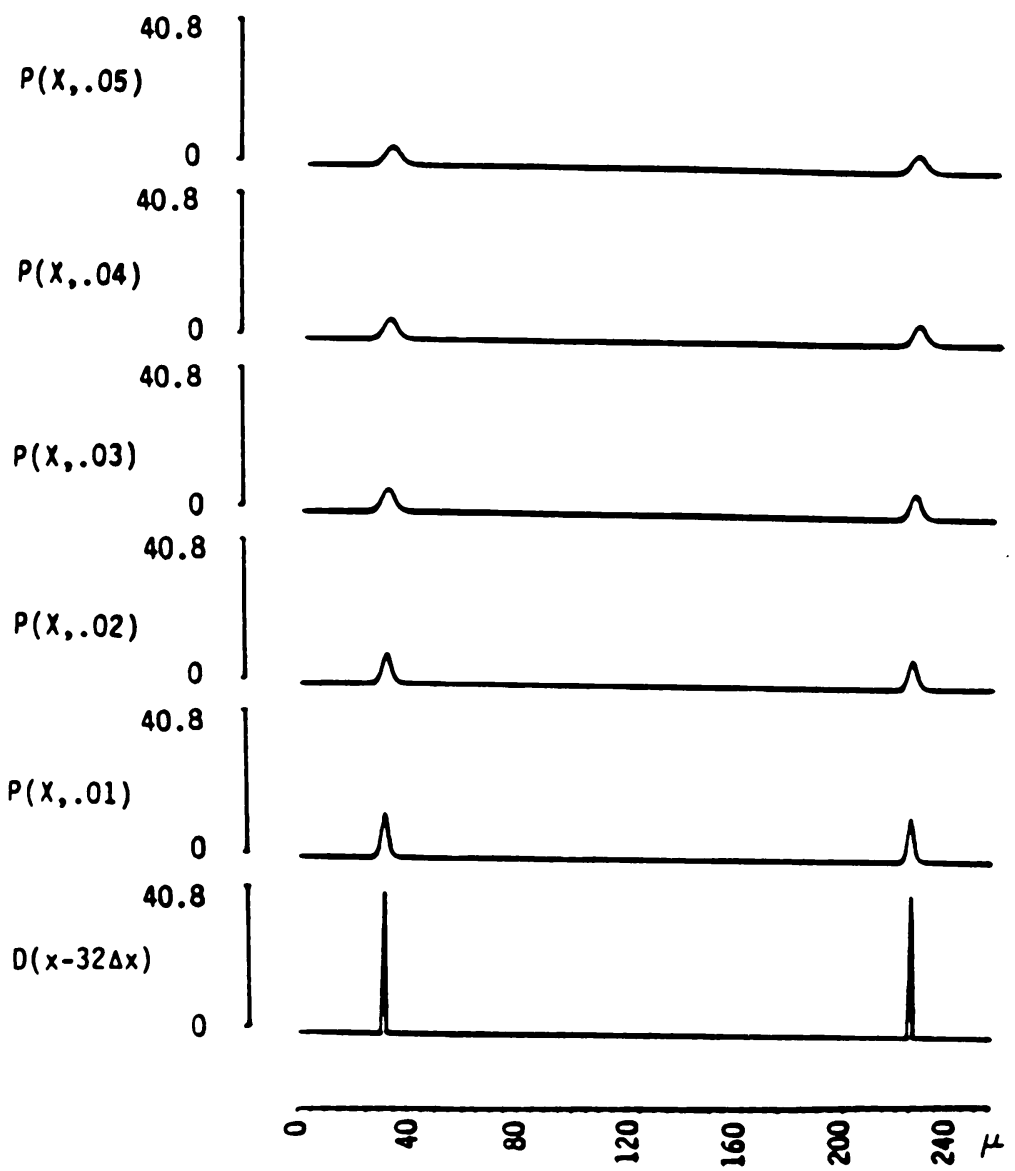

FIG. 3. Numerical solution using the conventionally discretized diffusion equation with a discretized delta function used as the initial field. The solution is displayed at 0.01 second time intervals. 


\section{REFERENCES}

[1] B. Compani-Tabrizi and R. G. Geyer, Differentiation by Fourier transformation and its connection with differentiation by finite differencing, Research Bulletin B85-2R or Technical Report TR85-15R, Marathon Oil Company, Research Center, Littleton, Colorado; Quart. Appl. Math. This issue

[2] E. Butkov, Mathematical Physics, Addison-Wesley (1968) 